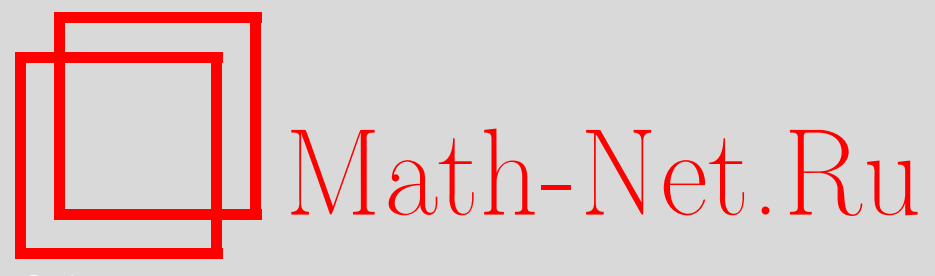

В. Н. Маклаков, Численное интегрирование краевых задач для нелинейных обыкновенных дифференциальных уравнений второго порядка произвольной структуры с использованием итерационных процедур, Вестн. Сам. гос. техн. ун-та. Сер. Физ.-мат. науки, 2016, номер 2, 354-365

DOI: https://doi.org/10.14498/vsgtu1481

Использование Общероссийского математического портала MathNet.Ru подразумевает, что вы прочитали и согласны с пользовательским соглашением

http: //www . mathnet.ru/rus/agreement

Параметры загрузки:

IP: 54.174 .149 .18

26 апреля 2023 г., 14:36:05

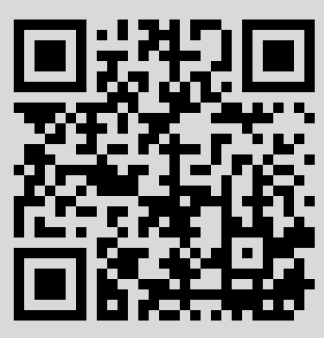


УДК 517.927.4:519.624.2

\title{
ЧИСЛЕННОЕ ИНТЕГРИРОВАНИЕ КРАЕВЫХ ЗАДАЧ ДЛЯ НЕЛИНЕЙНЫХ ОБЫКНОВЕННЫХ ДИФФЕРЕНЦИАЛЬНЫХ УРАВНЕНИЙ ВТОРОГО ПОРЯДКА ПРОИЗВОЛЬНОЙ СТРУКТУРЫ С ИСПОЛЬЗОВАНИЕМ ИТЕРАЦИОННЫХ ПРОЦЕДУР
}

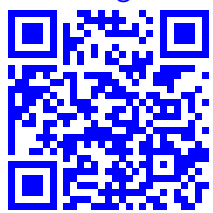

\section{В. Н. Маклаков}

Самарский государственный технический университет, Россия, 443100, Самара, ул. Молодогвардейская, 244.

\begin{abstract}
Аннотация
Предложена итерационная процедура численного интегрирования краевых задач для нелинейных обыкновенных дифференциальных уравнений второго порядка произвольной структуры. Исходное дифференциальное уравнение алгебраическими преобразованиями приведено к линейному неоднородному дифференциальному уравнению второго порядка с постоянными коэффициентами, правая часть которого представлена в виде линейной комбинации производных искомой функции вплоть до второй степени и исследуемого дифференциального уравнения произвольной структуры. При построении разностной краевой задачи были использованы многочлены Тейлора, что позволило отказаться от аппроксимации производных конечными разностями. Степень многочленов Тейлора может быть выбрана равной любому натуральному числу, большему или равному двум. Построенное линейное неоднородное дифференциальное уравнение имеет три произвольных коэффициента. Показано, что коэффициент при исходном дифференциальном уравнении произвольной структуры в правой части полученного неоднородного линейного дифференциального уравнения связан со сходимостью итерационной процедуры, а коэффициенты при производных искомой функции влияют на устойчивость разностной краевой задачи на каждой итерации. Теоретически установлены значения коэффициентов при производных искомой функции, обеспечивающие устойчивость разностной краевой задачи независимо от вида исходного уравнения. При выполнении численного эксперимента выявлено, что коэффициент, обеспечивающий сходимость итерационной процедуры, зависит от вида исходного дифференциального уравнения. Численный эксперимент показал, что увеличение степени используемого многочлена Тейлора приводит к уменьшению погрешности между точным и найденным численным решениями.
\end{abstract}

Ключевые слова: нелинейные обыкновенные дифференциальные уравнения, краевые задачи, численные методы, итерационные процедуры, ряды Тейлора.

(C) 2016 Самарский государственный технический университет.

Образец для цитирования

Мак лако в В. Н. Численное интегрирование краевых задач для нелинейных обыкновенных дифференциальных уравнений второго порядка произвольной структуры с использованием итерационных процедур // Вестн. Сам. гос. техн. ун-та. Сер. Физ.-мат. науки, 2016. T. 20, № 2. C. 354-365. doi: 10.14498/vsgtu1481.

\section{Сведения об авторе}

Владимир Николаевич Маклаков (к.ф.-м.н., доц.; makvo63@уandex.ru), доцент, каф. высшей математики и прикладной информатики. 
На основании предложенного в работе [1] метода интегрирования краевых задач для линейных обыкновенных дифференциальных уравнений второго порядка (ОДУ2) в [2] рассмотрена итерационная процедура нахождения численного решения краевых задач для нелинейных ОДУ2 специального вида

$$
r\left(x, u, u^{\prime}, u^{\prime \prime}\right) u^{\prime \prime}+p\left(x, u, u^{\prime}, u^{\prime \prime}\right) u^{\prime}+q\left(x, u, u^{\prime}, u^{\prime \prime}\right) u=f\left(x, u, u^{\prime}, u^{\prime \prime}\right),
$$

где $r\left(x, u, u^{\prime}, u^{\prime \prime}\right), p\left(x, u, u^{\prime}, u^{\prime \prime}\right),\left(x, u, u^{\prime}, u^{\prime \prime}\right), f\left(x, u, u^{\prime}, u^{\prime \prime}\right)$ - заданные функции, дифференцируемые по своим аргументам нужное число раз. Поставим целью построить итерационную процедуру численного интегрирования краевых задач для нелинейных ОДУ2 произвольной структуры.

1. Построение итерационной процедуры. Исследуем краевую дифференциальную задачу для ОДУ2 произвольной структуры

$$
\Psi\left(x, u, u^{\prime}, u^{\prime \prime}\right)=0, \quad x \in[a, b], \quad u(a)=\widetilde{u}_{0}, \quad u(b)=\widetilde{u}_{n},
$$

где отрезок $[a, b]$ - область интегрирования $D, \Psi\left(x, u, u^{\prime}, u^{\prime \prime}\right)$ - заданная функция, дифференцируемая по своим аргументам нужное число раз, $\widetilde{u}_{0}, \widetilde{u}_{n}-$ заданные числа.

На области $D$ зададим сетку $D_{h}$ равноотстоящими узлами, определяемыми значениями $x_{i}=x_{0}+i h, i=1,2, \ldots, n-1, x_{0}=a, x_{n}=b, h=(b-a) / n$.

Очевидно, ОДУ2 в краевой задаче (2) и следующее ОДУ2

$$
u^{\prime \prime}+p u^{\prime}+q u=u^{\prime \prime}+p u^{\prime}+q u+\lambda \Psi\left(x, u, u^{\prime}, u^{\prime \prime}\right),
$$

где $p, q, \lambda$ - постоянные величины, требующие дальнейшего уточнения, эквивалентны в смысле совпадения решений. Поэтому далее вместо (2) будем рассматривать краевую задачу вида

$$
u^{\prime \prime}+p u^{\prime}+q u=F\left(x, u, u^{\prime}, u^{\prime \prime}\right), \quad u(a)=\widetilde{u}_{0}, \quad u(b)=\widetilde{u}_{n},
$$

где $F\left(x, u, u^{\prime}, u^{\prime \prime}, \lambda\right)=u^{\prime \prime}+p u^{\prime}+q u+\lambda \Psi\left(x, u, u^{\prime}, u^{\prime \prime}\right)$ - известная функция при заданных значениях $p, q, \lambda$.

В дальнейшем для краткости примем для любой функции обозначение $\varphi\left(x_{i}\right)=\varphi_{i}$, где $x_{i}$ - узел сетки $D_{h}$.

При некотором фиксированном натуральном числе $k \geqslant 2$ для узла сетки $t_{i}, i=1,2, \ldots, n-1$, составим систему уравнений, состоящую из $(k+1)$-го уравнения. В качестве первого уравнения системы примем первые $k+1$ члена разложения в ряд Тейлора функции $u$ в узле $x_{i-1}$, в качестве второгов узле $x_{i+1}$. Дополнительно в систему внесём равенства, полученные дифференцированием по аргументу $x$ обеих частей уравнения в задаче $(3)$, так что

$$
\left(q u_{i}+p u_{i}^{\prime}+u_{i}^{\prime \prime}\right)^{(r)}=F_{i}^{(r)}
$$

где $r=0,1, \ldots, k-2$, которые запишем в узле $x_{i}$. Получим

$$
\left\{\begin{array}{l}
u_{i}-h u_{i}^{\prime}+\frac{h^{2}}{2 !} u_{i}^{\prime \prime}-\frac{h^{3}}{3 !} u_{i}^{\prime \prime \prime}+\cdots+(-1)^{k} \frac{h^{k}}{k !} u_{i}^{(k)}=u_{i-1} \\
u_{i}+h u_{i}^{\prime}+\frac{h^{2}}{2 !} u_{i}^{\prime \prime}+\frac{h^{3}}{3 !} u_{i}^{\prime \prime \prime}+\cdots+\frac{h^{k}}{k !} u_{i}^{(k)}=u_{i+1}, \\
q u_{i}+p u_{i}^{\prime}+u_{i}^{\prime \prime}=F_{i} \\
q u_{i}^{\prime}+p u_{i}^{\prime \prime}+u_{i}^{\prime \prime \prime}=F_{i}^{\prime} \\
\vdots \\
q u_{i}^{(k-2)}+p u_{i}^{(k-1)}+u_{i}^{(k)}=F_{i}^{(k-2)} .
\end{array}\right.
$$


В матричной форме система уравнений (4) имеет вид

$$
A^{k} V_{i}^{k}=G_{i}^{k}
$$

в обозначениях

$$
A^{k}=\left[\begin{array}{cccccc}
1 & -h & \frac{h^{2}}{2 !} & -\frac{h^{3}}{3 !} & \ldots & (-1)^{k} \frac{h^{k}}{k !} \\
1 & h & \frac{h^{2}}{2 !} & \frac{h^{3}}{3 !} & \ldots & \frac{h^{k}}{k !} \\
q & p & 1 & 0 & \ldots & 0 \\
0 & q & p & 1 & \ldots & 0 \\
\vdots & \vdots & \vdots & \vdots & \ddots & \vdots \\
0 & 0 & 0 & 0 & \ldots & 1
\end{array}\right], V_{i}^{k}=\left[\begin{array}{c}
u_{i} \\
u_{i}^{\prime} \\
u_{i}^{\prime \prime} \\
u_{i}^{\prime \prime} \\
\vdots \\
u_{i}^{(k)}
\end{array}\right], G_{i}^{k}=\left[\begin{array}{c}
u_{i-1} \\
u_{i+1} \\
F_{i} \\
F_{i}^{\prime} \\
\vdots \\
F_{i}^{(k-2)}
\end{array}\right]
$$

Элементы матрицы $A^{k}$ могут быть вычислены, следовательно, (4) является системой линейных алгебраических уравнений. Предполагая существование обратной матрицы $\left(A^{k}\right)^{-1}$ от матрицы $A^{k}$, найдём $V_{i}^{k}=\left(A^{k}\right)^{-1} G_{i}^{k}$, или в координатной форме:

$$
\begin{gathered}
u_{i}=a_{11}^{k} u_{i-1}+a_{12}^{k} u_{i+1}+a_{13}^{k} F_{i}+a_{14}^{k} F_{i}^{\prime}+\cdots+a_{1, k+1}^{k} F_{i}^{(k-2)}, \\
u_{i}^{\prime}=a_{21}^{k} u_{i-1}+a_{22}^{k} u_{i+1}+a_{23}^{k} F_{i}+a_{24}^{k} F_{i}^{\prime}+\cdots+a_{2, k+1}^{k} F_{i}^{(k-2)}, \\
u_{i}^{\prime \prime}=a_{31}^{k} u_{i-1}+a_{32}^{k} u_{i+1}+a_{33}^{k} F_{i}+a_{34}^{k} F_{i}^{\prime}+\cdots+a_{3, k+1}^{k} F_{i}^{(k-2)}, \\
\cdots \\
u_{i}^{(k)}=a_{k+1,1}^{k} u_{i-1}+a_{k+1,2}^{k} u_{i+1}+a_{k+1,3}^{k} F_{i}+\cdots+a_{k+1, k+1}^{k} F_{i}^{(k-2)},
\end{gathered}
$$

где под $a_{l m}^{k}, l, m=1,2, \ldots, k+1$, понимаем соответствующие элементы матрицы $\left(A^{k}\right)^{-1}$.

Составим систему уравнений, в которую внесём равенства (5) для всех $i=1,2, \ldots, n-1$ :

$$
\left\{\begin{array}{l}
-u_{1}+a_{12}^{k} u_{2}=-\sum_{m=0}^{k-2} a_{1, m+3}^{k} F_{1}^{(m)}-a_{11}^{k} \widetilde{u}_{0}, \\
a_{11}^{k} u_{1}-u_{2}+a_{12}^{k} u_{3}=-\sum_{m=0}^{k-2} a_{1, m+3}^{k} F_{2}^{(m)}, \\
a_{11}^{k} u_{2}-u_{3}+a_{12}^{k} u_{4}=-\sum_{m=0}^{k-2} a_{1, m+3}^{k} F_{3}^{(m)}, \\
\ldots \\
a_{11}^{k} u_{n-2}-u_{n-1}=-\sum_{m=0}^{k-2} a_{1, m+3}^{k} F_{n-1}^{(m)}-a_{12}^{k} \widetilde{u}_{n} .
\end{array}\right.
$$

Отметим, что (9) есть система разностных уравнений с постоянными коэффициентами для трёхточечного шаблона $x_{i-1}, x_{i}, x_{i+1}, i=1,2, \ldots, n-1$, 
при построении которой не использовались конечные разности для аппроксимации производных, как это было сделано при численном интегрировании краевых задач для обыкновенных дифференциальных уравнений в [3-6] и для ряда дифференциальных уравнений в частных производных в [5-8].

В матричной форме система (9) имеет вид

$$
B^{k} U=G^{k}
$$

в обозначениях

$$
\begin{gathered}
B^{k}=\left[\begin{array}{cccccc}
-1 & a_{12}^{k} & 0 & 0 & \ldots & 0 \\
a_{11}^{k} & -1 & a_{12}^{k} & 0 & \ldots & 0 \\
0 & a_{11}^{k} & -1 & a_{12}^{k} & \ldots & 0 \\
\vdots & \vdots & \vdots & \vdots & \ddots & \vdots \\
0 & 0 & 0 & 0 & \ldots & 1
\end{array}\right], \quad U=\left[\begin{array}{c}
u_{1} \\
u_{2} \\
u_{3} \\
\vdots \\
u_{n-1}
\end{array}\right], \\
G^{k}=G^{k}\left(U, U^{(1)}, \ldots, U^{(k)}\right)=\left[\begin{array}{c}
-\sum_{m=0}^{k-2} a_{1, m+3}^{k} F_{1}^{(m)}-a_{11}^{k} \widetilde{u}_{0} \\
-\sum_{m=0}^{k-2} a_{1, m+3}^{k} F_{2}^{(m)} \\
k-2 \\
-\sum_{m=0}^{k} a_{1, m+3}^{k} F_{3}^{(m)} \\
\vdots \\
-\sum_{m=0}^{k-2} a_{1, m+3}^{k} F_{n-1}^{(m)}-a_{12}^{k} \widetilde{u}_{n}
\end{array}\right] .
\end{gathered}
$$

В предположении существования обратной матрицы $\left(B^{k}\right)^{-1}$ от матрицы $B^{k}$ найдём $U=\left(B^{k}\right)^{-1} G^{k}$, или в развёрнутом виде

$$
U=\left(B^{k}\right)^{-1} G^{k}\left(U, U^{(1)}, \ldots, U^{(k)}\right)
$$

где $U^{(r)}=\left[\begin{array}{llll}u_{1}^{(r)} & u_{2}^{(r)} & \cdots & u_{n-1}^{(r)}\end{array}\right]^{\top}$ - обозначение вектор-столбца производных степени $r$ функции $u$ во внутренних узлах сетки $D_{h}$.

Матричное уравнение (10) наряду с (6)-(8) будем использовать при построении итерационной процедуры для нахождения приближённого решения задачи (3).

В качестве начального (нулевого) приближения $U_{0}$ примем расположенные на прямой $u=c x+d$, проведённой через точки $u(a)=\widetilde{u}_{0}, u(b)=\widetilde{u}_{n}$, значения функции $u$ во внутренних узла сетки. В этом случае, очевидно, значения производных нулевого приближения определим как

$$
U_{0}^{(1)}=\left[\begin{array}{llll}
c & c & \cdots & c
\end{array}\right]^{\top}, \quad U_{0}^{(r)}=\left[\begin{array}{llll}
0 & 0 & \cdots & 0
\end{array}\right]^{\top}, \quad r=2,3, \ldots, k .
$$

Подставим начальные приближения $U_{0}, U_{0}^{(r)}, r=1,2, \ldots, k$, в правые части матричного равенства (10), их вычислим и найденные значения примем в качестве первого приближения:

$$
U_{1}=\left(B^{k}\right)^{-1} G^{k}\left(U_{0}, U_{0}^{(1)}, \ldots, U_{0}^{(k)}\right) .
$$


Компоненты векторов первого приближения $U_{1}^{(r)}, r=1,2, \ldots, k$, вычислим по формулам (6)-(8) соответственно, в правые части которых подставим найденные значения вектора первого приближения $U_{1}$ и векторов начального приближения $U_{0}^{(r)}, r=1,2, \ldots, k$.

Найденные векторы первого приближения $U_{1}, U_{1}^{(r)}, r=1,2, \ldots, k$, вновь подставим в правую часть равенства (10), их вычислим и полученные значения примем в качестве второго приближения $U_{2}$ для искомой функции $u$. Для вычисления $U_{2}^{(r)}, r=1,2, \ldots, k$, воспользуемся формулами (6)-(8).

Описанная итерационная процедура может быть выполнена произвольное число раз при условии её сходимости по следующим формулам

$$
\begin{gathered}
U_{t}=\left(B^{k}\right)^{-1} G^{k}\left(U_{t-1}, U_{t-1}^{(1)}, \ldots, U_{t-1}^{(k)}\right), \\
u_{i t}^{(r)}=a_{(r+1) 1}^{k} u_{(i-1) t}+a_{(r+1) 2}^{k} u_{(i+1) t}+ \\
+\sum_{m=0}^{k-2} a_{(r+1)(m+3)}^{k} F_{i}^{(m)}, \quad i=1,2, \ldots, n-1, r=1,2, \ldots, k,
\end{gathered}
$$

где $t$ - номер итерации, $u_{i t}$ - компоненты вектора $U_{t}$.

2. Выбор постоянных. Постоянные величины $p, q$ выберем из требования обеспечения устойчивости задачи на каждой итерации.

Разностную краевую задачу с переменными коэффициентами

$$
a_{i} u_{i-1}+b_{i} u_{i}+c_{i} u_{i+1}=f_{i}, \quad u(a)=\widetilde{u}_{0}, \quad u(b)=\widetilde{u}_{n}, \quad i=1,2, \ldots, n-1,
$$

называют устойчивой [5], если она имеет единственное решение при произвольных $\widetilde{u}_{0}, \widetilde{u}_{n}, f_{1}, f_{2}, \ldots, f_{n-1}$ и имеет место оценка для норм

$$
\|u\| \leqslant C\|f\|,
$$

где $C$ - не зависящее от $h$ число, а в качестве нормы некоторого вектора $w=\left[\begin{array}{llll}w_{0} & w_{1} & \cdots & w_{n}\end{array}\right]^{\top}$ принято

$$
\|w\|=\max \left(\left|w_{0}\right|,\left|w_{1}\right|, \ldots,\left|w_{n}\right|\right) .
$$

В соответствии с (15) в неравенстве (14) положено

$$
u=\left[\begin{array}{llll}
u_{0} & u_{1} & \cdots & u_{n}
\end{array}\right]^{\top}, \quad f=\left[\begin{array}{llllll}
\widetilde{u}_{0} & \widetilde{u}_{n} & f_{1} & f_{2} & \cdots & f_{n-1}
\end{array}\right]^{\top} .
$$

Правомерность выбора нормы в виде (15) для разностной краевой задачи (13) обоснована в [5].

Разностную краевую задачу с постоянными коэффициентами

$$
a u_{i-1}+b u_{i}+c u_{i+1}=f_{i}, \quad u(a)=\widetilde{u}_{0}, \quad u(b)=\widetilde{u}_{n}, \quad i=1,2, \ldots, n-1
$$

называют хорошо обусловленной [5], если она при всех достаточно больших $n$ имеет единственное решение при произвольных $\widetilde{u}_{0}, \widetilde{u}_{n}, f_{1}, f_{2}, \ldots, f_{n-1}$ и если значения $u_{0}, u_{1}, \ldots, u_{n}$, образующие решение, удовлетворяют неравенству

$$
\left|u_{i}\right| \leqslant P \max \left(\left|\widetilde{u}_{0}\right|,\left|\widetilde{u}_{n}\right|,\left|f_{1}\right|,\left|f_{2}\right|, \ldots,\left|f_{n-1}\right|\right), \quad i=0,1, \ldots, n,
$$


где $P$ - не зависящее от $h$ число. Выполнение неравенства (17) означает, что погрешности, допущенные при задании $\widetilde{u}_{0}, \widetilde{u}_{n}, f_{1}, f_{2}, \ldots, f_{n-1}$, не накапливаются и не приводят к возрастающим с увеличением $n$ ошибкам [5].

Неравенство (14) и совокупность неравенств (17) эквивалентны в смысле совпадения оценок ввиду выбора нормы в форме (15) и в силу совпадения их правых частей; следовательно, хорошая обусловленность влечёт устойчивость на каждой итерации для рассматриваемой задачи.

Достаточным условием (критерием) хорошей обусловленности задачи (16) является выполнение неравенства [5]

$$
\frac{|b|-|a+c|}{|a|+|b|+|c|} \geqslant \Theta>0
$$

или

$$
|b|-|a+c|>0 \text {. }
$$

Критерий (18) для задачи (9) имеет вид

$$
1-\left|a_{11}^{k}+a_{12}^{k}\right|>0
$$

или

$$
\left|\operatorname{det} A^{k}\right|-\left|M_{11}^{k}+M_{12}^{k}\right|>0
$$

где $\operatorname{det} A^{k}$ - определитель матрицы $A^{k} ; M_{11}^{k}, M_{12}^{k}$ - алгебраические дополнения элементов, расположенных на пересечениях первой строки с первым и вторым столбцами, соответственно, матрицы $\left(A^{k}\right)^{\top}$.

Критерий (19) для задачи (9) при $k=2$ будет записан как

$$
\left|2 h-q h^{3}\right|-2 h>0
$$

откуда следуют решения $q<0$ и $q>4 / h^{2}$. Отбросим второе решение как зависящее от величины шага $h$. Критерий (19) при $k=3$ примет вид

$$
\left|2 h+\frac{\left(p^{2}-4 q\right) h^{3}}{3}+\frac{q^{2} h^{5}}{6}\right|-\left|2 h+\frac{\left(p^{2}-q\right) h^{3}}{3}\right|>0,
$$

откуда

$$
\left\{\begin{array} { l } 
{ 6 + ( p ^ { 2 } - q ) h ^ { 2 } > 0 , } \\
{ q ( q h ^ { 2 } - 6 ) > 0 , }
\end{array} \quad \left\{\begin{array}{l}
6+\left(p^{2}-q\right) h^{2}<0, \\
q\left(q h^{2}-6\right)<0 .
\end{array}\right.\right.
$$

Решением первой системы является $q<0$ для любого значения $p$, вторая система несовместна.

Нахождение аналитического решения неравенства (19) при $k>3$ оказалось довольно трудоёмким. В силу того, что элементы обратной матрицы $\left(A^{k}\right)^{-1}$ зависят только от параметров $p$ и $q$, с использованием персонального компьютера (ПК) была смоделирована процедура вычисления разности $\left|\operatorname{det} A^{k}\right|-\left|M_{11}^{k}+M_{12}^{k}\right|, k=4,5, \ldots, 8$, для различных $p$ и $q$. Для нескольких произвольных значений $p$ неравенство (19) обращалось в истину для ряда отрицательных значений $q$, в частности и для $q=-1$, которое и было, наряду с $p=1$, использовано при численном эксперименте, что гарантированно обеспечивало устойчивость на каждой итерации. 
3. Численный эксперимент. При выполнении численного эксперимента были использованы ОДУ2 специального вида (1)

$$
u u^{\prime \prime}-u^{\prime} u^{\prime}=1
$$

имеющее аналитическое решение

$$
u^{a}=\frac{1}{C_{1}} \operatorname{ch}\left(C_{1} x+C_{2}\right),
$$

с краевыми условиями

$$
u(-3)=5.10, \quad u(3)=7.71
$$

и ОДУ2 произвольной структуры

$$
e^{u^{\prime \prime}}-\left(u^{\prime \prime}\right)^{2}-x=0
$$

имеющее аналитическое решение в параметрической форме

$$
\left\{\begin{array}{l}
u^{a}(s)=\frac{1}{4} e^{2 s}(2 s-3)+\frac{1}{3} e^{s}\left(3 C_{1}-2 s^{3}+6 s-6\right)+\frac{1}{15} 4 s^{5}-C_{1} s^{2}+C_{2}, \\
x(s)=e^{s}-s^{2}
\end{array}\right.
$$

с краевыми условиями

$$
u(0)=1.11, \quad u(6)=7.43
$$

Общее решение ОДУ2 (20) и метод нахождения общего решения ОДУ2 (22) заимствованы из [9].

При выполнении численного эксперимента был использован метод перебора [6], или метод сканирования [10], согласно которому между некоторыми выбранными начальным $\lambda_{\text {str }}$ и конечным $\lambda_{\text {fin }}$ значениями с периодом дискретизации

$$
\Delta \lambda=\frac{\lambda_{\text {fin }}-\lambda_{\text {str }}}{r}
$$

вычислялись промежуточные значения

$$
\lambda_{m}=\lambda_{\mathrm{str}}+m \Delta \lambda, \quad m=0,1, \ldots, r
$$

и с каждым из них была реализована итерационная процедура (11), (12) для краевых задач $(20),(21)$ и $(22),(23)$.

Для каждого $\lambda_{m}$ при всех $k=2,3, \ldots, 8$ на каждой итерации с номером $t$ вычислялись следующие оценки погрешности на всём отрезке интегрирования:

1) $D(k, \lambda, t)=\frac{\sqrt{\sum_{i=1}^{n-1}\left(u_{i t}-u_{i}^{a}\right)^{2}}}{\sum_{i=1}^{n-1}\left|u_{i}^{a}\right|} \cdot 100 \%$ - суммарная оценка относительной погрешности на итерации с номером $t$, которую можно трактовать как некий аналог коэффициента вариации в статистике, характеризующий меру разброса в процентах [11]; величина $D$ отличается от коэффициента вариации тем, что стандартное отклонение заменено корнем квадратным из остаточной дисперсии [11]; 
2) $E(k, \lambda, t)=\sum_{i=1}^{n-1}\left|u_{i t}-u_{i}^{a}\right|$ - суммарная оценка абсолютной погрешности;

3) $H(k, \lambda, t)=\max \left|u_{i t}-u_{i}^{a}\right|, i=1,2, \ldots, n-1$ - максимальная оценка абсолютной погрешности;

4) $Q(k, \lambda, t)=\frac{\sqrt{\sum_{i=1}^{n-1}\left(u_{i t}-u_{i(t-1)}\right)^{2}}}{\sum_{i=1}^{n-1}\left|u_{i(t-1)}\right|} \cdot 100 \%$ - суммарная оценка относительной погрешности между приближёнными решениями на текущей и предыдущей итерациями с номерами $t$ и $(t-1)$ соответственно;

5) $R(k, \lambda, t)=\sum_{i=1}^{n-1}\left|u_{i t}-u_{i(t-1)}\right|$ - суммарная оценка абсолютной погрешности между текущей и предыдущей итерациями;

6) $S(k, \lambda, t)=\max \left|u_{i t}-u_{i(t-1)}\right|, i=1,2, \ldots, n-1$ - максимальная оценка абсолютной погрешности между текущей и предыдущей итерациями.

Приведём некоторые результаты, выявленные сканированием по параметру $\lambda$ в задачах (20), (21) и (22), (23).

Расчётным путём для рассматриваемых задач установлено, что при фиксированных $k$ и $\lambda$ оценки погрешностей $D, E, H$ между точным и приближённым решениями с увеличением номера итерации $t$ изменялись практически синхронно (росли или убывали одновременно). Аналогичная ситуация складывается с оценками погрешностей $Q, R, S$ между приближёнными решениями на текущей и предыдущей итерациями.

Для каждого набора чисел $k$ и $\lambda$ при отсутствии переполнения ПК peaлизация итерационной процедуры прекращалась на итерации с номером $t_{\mathrm{ust}}$ при выполнения условия

$$
D\left(k, \lambda, t_{\mathrm{ust}}\right)-D\left(k, \lambda, t_{\mathrm{ust}-1}\right) \leqslant 10^{-15} .
$$

В расчётах для обеих задач принято $n=20, h=0.3$. В итоге предварительного грубого сканирования области $\lambda \in[-10 ; 10]$ с периодом дискретизации $\Delta \lambda=5 \cdot 10^{-2}$ для обеих задач были найдены интервалы изменения параметра $\lambda$, в которых выполнено условие (24), а далее было положено $\Delta \lambda=$ $=4.5 \cdot 10^{-3}$ в задаче $(20),(21)$ и $\Delta \lambda=5.5 \cdot 10^{-3}$ в задаче $(22),(23)$ для итогового сканирования.

Для дискретно заданных функций $D(t), Q(t)$ численным экспериментом установлено, что в интервале изменения параметра $\lambda$ для всех $k=2,3, \ldots, 8$ :

a) график функции $D(t)$ имеет один локальный минимум в точке $t_{\min }$ и одну точку перегиба;

б) график функции $D(t)$ имеет горизонтальную асимптоту $D=D_{\text {ust }}=$ $=D\left(t_{\mathrm{ust}}\right)$;

в) точки $t_{\min }, t_{\text {ust }}$ смещаются вправо при уменьшении $|\lambda|$;

г) при фиксированном $k$ погрешность $D_{\min }=D\left(t_{\min }\right)$ практически не зависит от $\lambda$;

д) функция $Q(t)$ оказалась невозрастающей, её график имеет горизонтальную асимптоту $Q=0$;

е) при фиксированном $k$ модуль производной $\left|Q(t)^{\prime}\right|$ уменьшается при уменьшении $|\lambda|$.

Результаты расчётов для задачи (20), (21) приведены в табл. 1, для задачи $(22),(23)$ - в табл. 2. В табл. 1,2 принято $Q_{\min }=Q\left(t_{\min }\right), Q_{\text {ust }}=Q\left(t_{\text {ust }}\right)$; в качестве $t_{\min }$ указано наименьшее значение из всех вычисленных $t_{\min }(\lambda)$ 
Таблица 1

Значения погрешностей краевой задачи (20), (21) [The values of the errors for the boundary value problem (20), (21)]

\begin{tabular}{c|c|c|c|c|c|c|c}
\hline$k$ & $\lambda$ & $t_{\min }$ & $D_{\min }, \%$ & $Q_{\min }, \%$ & $t_{\text {ust }}$ & $D_{\text {ust }}, \%$ & $Q_{\text {ust }}, \%$ \\
\hline 2 & -0.3240 & 34 & $9.74 \cdot 10^{-4}$ & $2.56 \cdot 10^{-4}$ & 37 & $1.19 \cdot 10^{-3}$ & $2.49 \cdot 10^{-5}$ \\
3 & -0.2970 & 39 & $3.42 \cdot 10^{-4}$ & $1.33 \cdot 10^{-4}$ & 42 & $3.73 \cdot 10^{-4}$ & $7.21 \cdot 10^{-6}$ \\
4 & -0.2790 & 51 & $6.94 \cdot 10^{-7}$ & $1.69 \cdot 10^{-7}$ & 76 & $7.19 \cdot 10^{-7}$ & $9.94 \cdot 10^{-9}$ \\
5 & -0.2745 & 53 & $6.79 \cdot 10^{-7}$ & $1.93 \cdot 10^{-7}$ & 83 & $6.82 \cdot 10^{-7}$ & $7.14 \cdot 10^{-10}$ \\
6 & -0.2700 & 49 & $6.74 \cdot 10^{-7}$ & $7.47 \cdot 10^{-8}$ & 87 & $6.94 \cdot 10^{-7}$ & $7.24 \cdot 10^{-11}$ \\
7 & -0.2700 & 49 & $6.74 \cdot 10^{-7}$ & $7.49 \cdot 10^{-8}$ & 87 & $6.94 \cdot 10^{-7}$ & $4.48 \cdot 10^{-11}$ \\
8 & -0.2700 & 49 & $6.74 \cdot 10^{-7}$ & $7.46 \cdot 10^{-8}$ & 88 & $6.94 \cdot 10^{-7}$ & $2.32 \cdot 10^{-11}$ \\
\hline
\end{tabular}

Таблица 2

Значения погрешностей краевой задачи (22), (23) [The values of the errors for the boundary value problem $(22),(23)]$

\begin{tabular}{c|c|c|c|c|c|c|c}
\hline$k$ & $\lambda$ & $t_{\min }$ & $D_{\min }, \%$ & $Q_{\min }, \%$ & $t_{\text {ust }}$ & $D_{\text {ust }}, \%$ & $Q_{\text {ust }}, \%$ \\
\hline 2 & -0.3960 & 29 & $6.13 \cdot 10^{-2}$ & $1.71 \cdot 10^{-2}$ & 66 & $6.22 \cdot 10^{-2}$ & $4.19 \cdot 10^{-6}$ \\
3 & -0.3740 & 30 & $9.78 \cdot 10^{-3}$ & $1.92 \cdot 10^{-2}$ & 79 & $6.22 \cdot 10^{-2}$ & $3.71 \cdot 10^{-7}$ \\
4 & -0.3630 & 67 & $9.69 \cdot 10^{-5}$ & $6.78 \cdot 10^{-6}$ & 98 & $9.78 \cdot 10^{-5}$ & $1.12 \cdot 10^{-9}$ \\
5 & -0.3575 & 71 & $9.71 \cdot 10^{-5}$ & $3.64 \cdot 10^{-6}$ & 102 & $9.78 \cdot 10^{-5}$ & $3.45 \cdot 10^{-10}$ \\
6 & -0.3465 & 69 & $8.18 \cdot 10^{-6}$ & $2.89 \cdot 10^{-6}$ & 105 & $1.11 \cdot 10^{-5}$ & $2.54 \cdot 10^{-10}$ \\
7 & -0.3465 & 69 & $8.18 \cdot 10^{-6}$ & $3.53 \cdot 10^{-6}$ & 111 & $1.11 \cdot 10^{-5}$ & $1.61 \cdot 10^{-10}$ \\
8 & -0.3465 & 69 & $8.17 \cdot 10^{-6}$ & $4.20 \cdot 10^{-6}$ & 113 & $1.15 \cdot 10^{-5}$ & $6.97 \cdot 10^{-11}$ \\
\hline
\end{tabular}

при фиксированном $k$, что соответствует левой границе интервала изменения $\lambda$. Правая граница упомянутого интервала оказалась меньше нуля на 3-4 периода дискретизации $\Delta \lambda$ для обеих задач.

Данные табл. 1, 2 свидетельствуют о том, что, как и в работах $[1,2,12]$, имеет место уменьшение погрешностей с увеличением числа $k$.

Анализ итогов численного эксперимента позволяет сделать вывод, что параметр $\lambda$, как оказалось, являющийся «ответственным» за сходимость итерационной процедуры, можно трактовать как некую характеристику «противовеса», уравнивающую «вес» влияния слагаемых $u^{\prime \prime}+p u^{\prime}+q u$ и $\lambda \Psi\left(x, u, u^{\prime}, u^{\prime \prime}\right)$ друг на друга в правой части задачи (3). Теоретическое обоснование выбора значения параметра $\lambda$ требует дополнительного исследования. Также требуют дальнейшей теоретической проработки вопросы, связанные с определением момента достижения заданной точности.

4. Выводы. Предложена итерационная процедура численного интегрирования краевых задач для обыкновенных дифференциальных уравнений второго порядка произвольной структуры. Опытным путём выявлены некоторые закономерности влияния значения параметра $\lambda$ на сходимость. Теоретически установлены значения коэффициентов, обеспечивающие устойчивость на каждой итерации независимо от вида исходного уравнения произвольной структуры. Численным экспериментом показано, что использование многочленов Тейлора произвольных степеней вместо конечных разностей при аппроксимации производных приводит к уменьшению погрешностей между точным и найденным численным решениями.

\section{ORCID}

Владимир Николаевич Маклаков: http://orcid.org/0000-0003-1644-7424 


\section{БИБЛИОГРАФИЧЕСКИЙ СПИСОК}

1. Радченко В. П., Усов А. А. Модификация сеточных методов решения линейных дифференциальных уравнений с переменными коэффициентами на основе тейлоровских разложений // Вестн. Сам. гос. техн. ун-та. Сер. Физ.-мат. науки, 2008. №2(17). C. 60-65. doi: $10.14498 / v s g t u 646$.

2. Маклаков В. Н., Усов А. А. Численное интегрирование матричным методом краевых задач для нелинейных обыкновенных дифференциальных уравнений второго порядка с использованием итерационных процедур / Труды девлтой Всероссийской научной конференции с международным участием. Часть 3 / Математическое моделирование и краевые задачи. Самара: СамГТУ, 2013. С. 35-42.

3. Lentini M., Pereyra V. A Variable Order Finite Difference Method for Nonlinear Multipoint Boundary Value Problems // Mathematics of Computation, 1974. vol. 28, no. 128. pp. 9811003. doi: $10.2307 / 2005360$.

4. Keller H. B. Numerical Solution of Boundary Value Problems for Ordinary Differential Equations: Survey and Some Resent Results on Difference Methods / Numerical Solution of Boundary Value Problems for Ordinary Differential Equations. New York: Academic Press, 1975. pp. 27-88. doi : 10.1016/b978-0-12-068660-5.50007-7.

5. Годунов С. К., Рябенький В. С. Разностные схемы. М.: Наука, 1977. 439 с.

6. Формалеев В. Ф., Ревизников Д. Л. Численные методы. М.: Физматлит, 2004. 400 с.

7. Boutayeb A., Chetouani A. Global extrapolations of numerical methods for solving a parabolic problem with non local boundary conditions // International Journal of Computer Mathematics, 2003. vol.80, no.6. pp. 789-797. doi : 10.1080/0020716021000039209.

8. Boutayeb A., Chetouani A. A Numerical Comparison of Different Methods Applied to the Solution of Problems with Non Local Boundary Conditions // Applied Mathematical Sciences, 2007. vol. 1, no. 44. pp. 2173-2185.

9. Васильков Ю. В., Василькова Н. Н. Компьютерные технологии вычислений в математическом моделировании. М.: Финансы и статистика, 1999. 255 с.

10. Камке Э. Справочник по обыкновенным дифференциальным уравнениям. М.: Наука, 1976. $576 \mathrm{c}$.

11. Закс Л. Статистическое оченивание. М.: Статистика, 1976. 598 с.

12. Маклаков В. Н. Итерационный метод численного интегрирования краевых задач для систем нелинейных обыкновенных дифференциальных уравнений второго порядка / Труды десятой Всероссийской научной конферениии с международным участием. Часть 3 / Математическое моделирование и краевые задачи. Самара: СамГТУ, 2016. C. $50-58$.

Поступила в редакцию 09/III/2016; в окончательном варианте - 15/IV /2016; принята в печать $-27 / \mathrm{V} / 2016$. 
Vestn. Samar. Gos. Techn. Un-ta. Ser. Fiz.-mat. nauki

[J. Samara State Tech. Univ., Ser. Phys. \& Math. Sci.], 2016, vol. 20, no. 2, pp. $354-365$

ISSN: 2310-7081 (online), 1991-8615 (print)

doi: http://dx.doi.org/10.14498/vsgtu1481

MSC: 34B99

\title{
NUMERICAL INTEGRATION OF THE BOUNDARY VALUE PROBLEMS FOR THE SECOND ORDER NONLINEAR ORDINARY DIFFERENTIAL EQUATIONS OF AN ARBITRARY STRUCTURE USING AN ITERATIVE PROCEDURE
}

\section{N. Maklakov}

Samara State Technical University,

244, Molodogvardeyskaya st., Samara, 443100, Russian Federation.

\begin{abstract}
An iterative procedure for numerical integration of boundary-value problems for nonlinear ordinary differential equations of the second order of arbitrary structure is suggested. The initial differential equation by algebraic transformation can be written as a linear inhomogeneous differential equation of the second order with constant coefficients; the right part of which is represented as a linear combination of the derivatives of the required function up to the second order and a differential equation of arbitrary structure under study. Taylor polynomials were used in the construction of the difference boundary value problem. This allowed to abandon the approximation of derivatives by finite differences. The degree of Taylor polynomials can be chosen as any natural number greater than or equal to two. Obtained inhomogeneous linear differential equation has three arbitrary coefficients. It is shown that the coefficient at the initial differential equations of any structure on the right side of the obtained non-homogeneous linear differential equation is associated with the convergence of the iterative procedure; and the coefficients at the derivatives of the required function affect the stability of difference boundary value problem at each iteration. The values of coefficients at the derivatives of the required function which ensure the stability of difference boundary value problem regardless of the type of the initial equation are theoretically set up. Numerical experiment showed that the coefficient providing the convergence of the iterative procedure depends on the type of the initial differential equation. Numerical experiments showed that the increase in the degree of the Taylor polynomial reduces the error between the exact and the obtained approximate solutions.
\end{abstract}

Keywords: nonlinear ordinary differential equations, boundary value problems, numerical methods, iterative procedures, Taylor series.

(C) 2016 Samara State Technical University.

Please cite this article in press as:

Maklakov V. N. Numerical integration of the boundary value problems for the second order nonlinear ordinary differential equations of an arbitrary structure using an iterative procedure, Vestn. Samar. Gos. Tekhn. Univ., Ser. Fiz.-Mat. Nauki [J. Samara State Tech. Univ., Ser. Phys. \& Math. Sci.], 2016, vol. 20, no. 2, pp. 354-365. doi: 10.14498/vsgtu1481. (In Russian) Author Details:

Vladimir N. Maklakov (Cand. Phys. \& Math. Sci.), Associate Professor, Dept. of Higher Mathematics and Applied Informatics. 


\section{ORCID}

Vladimir N. Maklakov: http://orcid.org/0000-0003-1644-7424

\section{REFERENCES}

1. Radchenko V. P., Usov A. A. Modified grid method for solving linear differential equation equipped with variable coefficients based on Taylor series, Vestn. Samar. Gos. Tekhn. Univ. Ser. Fiz.-Mat. Nauki [J. Samara State Tech. Univ., Ser. Phys. \& Math. Sci.], 2008, no. 2(17), pp. 60-65 (In Russian). doi: 10.14498/vsgtu646.

2. Maklakov V. N., Usov A. A. Numerical integration of boundary value problems for second order nonlinear ordinary differential equations by the matrix method using iterative procedures, Proceedings of the Ninth All-Russian Scientific Conference with international participation. Part 3, Matem. Mod. Kraev. Zadachi. Samara, Samara State Technical Univ., 2013, pp. 35-42 (In Russian).

3. Lentini M., Pereyra V. A Variable Order Finite Difference Method for Nonlinear Multipoint Boundary Value Problems, Mathematics of Computation, 1974, vol.28, no. 128, pp. 9811003. doi : $10.2307 / 2005360$.

4. Keller H. B. Numerical Solution of Boundary Value Problems for Ordinary Differential Equations: Survey and Some Resent Results on Difference Methods, Numerical Solution of Boundary Value Problems for Ordinary Differential Equations. New York, Academic Press, 1975, pp. 27-88. doi : 10.1016/b978-0-12-068660-5.50007-7.

5. Godunov S. K., Ryaben'kii V. S. Raznostnye skhemy [Difference Scheme]. Moscow, Nauka, 1977, 439 pp. (In Russian)

6. Formaleev V. F., Reviznikov D. L. Chislennye metody [Numerical methods]. Moscow, Fizmatlit, 2004, 400 pp. (In Russian)

7. Boutayeb A., Chetouani A. Global extrapolations of numerical methods for solving a parabolic problem with non local boundary conditions, International Journal of Computer Mathematics, 2003, vol.80, no. 6, pp. 789-797. doi:10.1080/0020716021000039209.

8. Boutayeb A., Chetouani A. A Numerical Comparison of Different Methods Applied to the Solution of Problems with Non Local Boundary Conditions, Applied Mathematical Sciences, 2007, vol. 1, no. 44, pp. 2173-2185.

9. Vasil'kov Yu. V., Vasil'kova N. N. Komp'iuternye tekhnologii vychislenii v matematicheskom modelirovanii [Computer technology computing in mathematical modeling]. Moscow, Finansy i statistika, 1999, 255 pp. (In Russian)

10. Kamke E. Spravochnik po obyknovennym differentsial'nym uravneniiam [Handbook on Ordinary Differential Equations]. Moscow, Nauka, 1976, 576 pp. (In Russian)

11. Zaks L. Statisticheskoe otsenivanie [Statistical estimation]. Moscow, Statistika, 1976, 598 pp. (In Russian)

12. Maklakov V. N. An iterative method for the numerical integration of boundary value problems for systems of the second order nonlinear ordinary differential equations, Proceedings of the Tenth All-Russian Scientific Conference with international participation. Part 3, Matem. Mod. Kraev. Zadachi. Samara, Samara State Technical Univ., 2016, pp. 50-58 (In Russian).

Received 09/III/2016;

received in revised form $15 / \mathrm{IV} / 2016$;

accepted 27/V/2016. 